\title{
Restricted divisor sums
}

\author{
by \\ Kevin A. Broughan (Hamilton)
}

1. Introduction. There is a body of work in the literature on various restricted sums of the number of divisors of an integer function including that described in $[2-9,11]$ and summarised in Section 2 below. In this paper asymptotic expressions are derived for sums of the form

$$
\sum_{1 \leq n \leq x} d_{\alpha}(f(n))
$$

where the function $f(n)$ is $n, n^{2}$ or $n^{2}+n+(p+1) / 4$, where $p \equiv 3 \bmod 4$ is a rational prime, and where

for real $\alpha \geq 1$.

$$
d_{\alpha}(n)=\#\{d: d \mid n \text { and } 1 \leq d \leq \alpha\}
$$

Motivation for considering these sums comes from an expression which is derived for the class number of a quadratic field with discriminant $-p$, in terms of a certain restricted divisor sum. This sum is currently too difficult to estimate, in that the restrictions on divisors depend on the summation variable $n$.

In deriving asymptotic expressions for the sum

$$
\sum_{1 \leq n \leq x} d_{\alpha}\left(n^{2}\right)
$$

it is natural to introduce two so-called integer square root functions $r_{+}(n)$ and $r_{-}(n)$. Both are multiplicative and take integer values. Their Dirichlet series are expressible in a compact rational form in terms of the Riemann zeta function.

2. The class number. Define, for positive integral $n$ and real $a, b$ with $a<b$, the restricted divisor function:

$$
d(n,(a, b))=\#\{d: d \mid n \text { and } a<d<b\} .
$$

2000 Mathematics Subject Classification: 11A25, 11A51, 11M06, 11N25, 11N37.

Key words and phrases: Dirichlet series, divisor sum, class number, integer square root. 
THEOREM 2.1. Let $p$ be a rational prime with $p>3$ and $p \equiv 3 \bmod 4$. Then the class number for quadratic forms with discriminant $-p$ can be expressed in the form

$$
\begin{aligned}
& h(-p) \\
& =1+2 \sum_{0 \leq n \leq \frac{1}{2} \sqrt{\frac{p}{3}}-1} d\left(n^{2}+n+\frac{p+1}{4},\left(2 n+1, \sqrt{n^{2}+n+\frac{p+1}{4}}\right)\right) .
\end{aligned}
$$

Proof. If $f(x, y)=a x^{2}+b x y+c y^{2}$ in $\mathbb{Z}[x, y]$ is a quadratic form then it is primitive if

(i) $0 \leq b \leq a=c$ or

(ii) $-a<b<a<c$ or

(iii) $0<b=a<c$.

The discriminant is

$$
-p=b^{2}-4 a c
$$

so $b \neq 0$.

Corresponding to each triple $(a, b, c)$ satisfying (i), (ii) or (iii) there is a form, and different triples correspond to inequivalent forms.

In case (i) $p=(2 a)^{2}-b^{2}=(2 a+b)(2 a-b)$ so $2 a+b=p$ and $2 a-b=1$. Therefore $a=(p+1) / 4$ and $b=(p-1) / 2$ so there is one solution at most. But $b \leq a$ implies $p \leq 3$, so there are no solutions in case (i).

In case (ii) assume $1 \leq b \leq a<c$, since if $b$ is a solution so is $-b$ and vice versa.

By (3), $b$ is odd. Since also $p \equiv 3 \bmod 4$,

$$
a c=\frac{b^{2}+p}{4}
$$

is an integer. Hence $a \mid\left(b^{2}+p\right) / 4$ and $a<c$ therefore $a<\sqrt{\left(b^{2}+p\right) / 4}$ hence $4 a^{2}-p<b^{2}<a^{2}$ so $a<\sqrt{p / 3}$ and $b \leq \sqrt{p / 3}-1$.

Similarly $a<c$ if and only if $a<\sqrt{\left(b^{2}+p\right) / 4}$.

In case (iii), $1 \leq b=a<c$ so $p=a(4 c-a)$. The relation $a=p$ is impossible, since then $4 c-a=1$ and $c=(p+1) / 4<p=a$.

If $a=1$ and $4 c-a=p$ we obtain $c=(p+1) / 4$, leading to the so-called principal solution

$$
(a, b, c)=(1,1,(p+1) / 4) .
$$

Conversely, if $a \mid\left(p+b^{2}\right) / 4$ and $b<a<\sqrt{\left(p+b^{2}\right) / 4}$ with $1 \leq b \leq \sqrt{p / 3}-1$, then $-p=b^{2}-4 a c$ and $1 \leq b<a<c$.

For each odd value of $b$ satisfying $1 \leq b \leq \sqrt{p / 3}-1$ we count the number of divisors $a$ of $\left(b^{2}+p\right) / 4$ satisfying $b<a<\sqrt{\left(b^{2}+p\right) / 4}$, double to account 
for each solution $(a,-b, c)$, and add 1 for the principal solution to obtain

$$
h(-p)=1+2 \sum_{\substack{1 \leq b \leq \sqrt{p / 3}-1 \\ b \text { odd }}} d\left(\frac{p+b^{2}}{4},\left(b, \sqrt{\frac{p+b^{2}}{4}}\right)\right) .
$$

Finally, let $b=2 n+1$ to obtain formula (2).

Corollary 2.1. The class number $h(-p)$ is odd.

Corollary 2.2. For all primes $p \equiv 3 \bmod 4$ with $p>3$,

$$
h(-p) \geq d\left(\frac{1+p}{4}\right)-1
$$

since $(1+p) / 4$ is never a square.

COROLlary 2.3. The following upper bound is an immediate consequence:

$$
h(-p) \leq\left(1-\frac{1}{2} \sqrt{\frac{p}{3}}\right)+\sum_{0 \leq n \leq \frac{1}{2} \sqrt{\frac{p}{3}}-1} d\left(n^{2}+n+\frac{p+1}{4}\right) .
$$

EXAMPLE 2.1. If $p=59$ a set of inequivalent representatives is $\{(1,1,15)$, $(3,1,5),(3,-1,5)\}$ and $h(-59)=3$.

If $p=151$ then

$d(38,(1,6])=1, \quad d(40,(3,6])=2, \quad d(44,(5,6])=0, \quad d(50,(7,7])=0$, so $h(-151)=1+2(1+2)=7$.

3. Existing results. To begin with there is Dirichlet's famous divisor sum theorem of 1850, and its improvements due to Voronoi in 1903 and van der Corput in 1922. We have

$$
D(x)=\sum_{n \leq x} d(n)=x \log x+(2 \gamma-1) x+O(f(x))
$$

where a more recent improvement is $f(x)=x^{a}$ with $a=12 / 37+\varepsilon$, due to Kolesnik [7], and $f(x)=x^{a} \log ^{b} x$ with $a=23 / 73$ and $b=461 / 146$, due to Huxley [5], is the best known published result.

In 1952 Erdős [2] showed that if $f$ is a polynomial with integer coefficients, then there are positive constants $A_{1}$ and $A_{2}$ such that

$$
A_{1} x \log x<\sum_{n \leq x} d(f(n))<A_{2} x \log x
$$

where the constants $A_{i}$ depend on the coefficients (and hence also the degree) of $f$. 
In [12] Scourfield quoting a result of Bellman-Shapiro states that if $f$ is an irreducible quadratic polynomial with integral coefficients, then

$$
\sum_{n \leq x} d(f(n))=A x \log x+O(x \log \log x),
$$

where the constant $A$ depends on the coefficients of $f$. This result was improved by McKee in [8-10] who derived an error bound of $O(x)$.

In 1963 Hooley [4] considered the special case of $\sum_{n \leq x} d\left(n^{2}+a\right)$ and found asymptotic expressions for the cases $a=-k^{2}$ and $a \neq-k^{2}$.

Other results for restricted divisor sums include divisors in short intervals $[3,11]$ and a number of results for divisors in arithmetic progressions. The monograph [3] covers in depth a range of related concepts.

In this article we begin the task of analysing the class number divisor sum derived in Theorem 2.1 above by looking at the sums where the divisors are restricted in size, independent of the summation range. This is done first for $f(n)=n$ then $f(n)=n^{2}$ and finally $f(n)=n^{2}+n+(p+1) / 4$. In each case asymptotic expressions are derived.

\section{Sums restricted by divisor size}

TheOREM 4.1. Let $1 \leq \alpha \leq x$ be real numbers. Then

$$
D(x, \alpha)=\sum_{1 \leq n \leq x} d_{\alpha}(n)=x \log \alpha+x \gamma+O(x / \alpha)+O(\alpha) .
$$

Proof. Simply count the lattice points below the curve $u v=x$ and above the interval $[1, \alpha]$ (see for example [1]):

$$
\begin{aligned}
D(x, \alpha) & =\sum_{1 \leq j \leq \alpha}\left\lfloor\frac{x}{j}\right\rfloor=\sum_{1 \leq j \leq \alpha}\left(\frac{x}{j}-\left\{\frac{x}{j}\right\}\right) \\
& =x(\log \alpha+\gamma+O(1 / \alpha))+O(\alpha) \\
& =x \log \alpha+x \gamma+O(x / \alpha)+O(\alpha)
\end{aligned}
$$

where $\gamma$ is Euler's constant.

5. Integer square roots. In this section we will derive an asymptotic expression for the restricted divisor sum

$$
D_{2}(x, \alpha)=\sum_{1 \leq n \leq x} d_{\alpha}\left(n^{2}\right)
$$

by first expressing it in terms of the integer square root function $r_{+}(n)$ defined as follows: If $n$ is a positive integer, $r_{+}(n) \mid n$ and $n \mid r_{+}(n)^{2}$, and if $d$ is such that $d \mid n$ with $n \mid d^{2}$ then $r_{+}(n) \mid d$. This defines $r_{+}(n)$ uniquely. 
Let $r_{-}(n)=n / r_{+}(n)$. Then if

$$
n=\prod_{i=1}^{m} p_{i}^{\alpha_{i}}
$$

we have

$$
r_{+}(n)=\prod_{i=1}^{m} p_{i}^{\left\lceil\alpha_{i} / 2\right\rceil} \quad \text { and } \quad r_{-}(n)=\prod_{i=1}^{m} p_{i}^{\left\lfloor\alpha_{i} / 2\right\rfloor} .
$$

Note that $r_{+}(n)^{2}=n$ if and only if $n$ is a perfect square, that for all primes $p$, if $p \mid n$ then $p \mid r_{+}(n), r_{+}(n) r_{-}(n)=n$ and $\sqrt{n} \leq r_{+}(n) \leq n$. Also $\left(r_{+}(n), r_{+}(m)\right)=r_{+}((n, m))$ where $(n, m)$ is the greatest common divisor. Finally, both $r_{+}$and $r_{-}$are multiplicative, but not completely multiplicative.

We will develop four Dirichlet series for these functions:

$$
\psi_{ \pm}(s)=\sum_{n=1}^{\infty} \frac{r_{ \pm}(n)}{n^{s}}, \quad \phi_{ \pm}(s)=\sum_{n=1}^{\infty} \frac{1 / r_{ \pm}(n)}{n^{s}} .
$$

TheOREm 5.1. For $\sigma=\Re(s)$ sufficiently large, the Dirichlet series satisfy the following:

$$
\begin{array}{ll}
\phi_{+}(s)=\frac{\zeta(2 s+1) \zeta(s+1)}{\zeta(2 s+2)} & (\sigma>0), \\
\phi_{-}(s)=\frac{\zeta(2 s+1) \zeta(s)}{\zeta(2 s)} & (\sigma>1), \\
\psi_{+}(s)=\frac{\zeta(2 s-1) \zeta(s-1)}{\zeta(2 s-2)} & (\sigma>2), \\
\psi_{-}(s)=\frac{\zeta(2 s-1) \zeta(s)}{\zeta(2 s)} & (\sigma>1) .
\end{array}
$$

Proof. If $p$ is a prime then $r_{+}\left(p^{\alpha}\right)=p^{\lceil\alpha / 2\rceil}$ so

$$
\begin{aligned}
\phi_{+}(s) & =\prod_{p}\left(1+\frac{1}{r_{+}(p) p^{s}}+\frac{1}{r_{+}\left(p^{2}\right) p^{2 s}}+\ldots\right) \\
& =\prod_{p}\left(1+\frac{1}{p p^{s}}+\frac{1}{p p^{2 s}}+\frac{1}{p^{2} p^{3 s}}+\frac{1}{p^{2} p^{4 s}}+\ldots\right) \\
& =\prod_{p}\left(1+\frac{p^{s}}{\left(p p^{2 s}\right)^{1}}+\left(\frac{1}{p p^{2 s}}\right)^{1}+\frac{p^{s}}{\left(p p^{2 s}\right)^{2}}+\left(\frac{1}{p p^{2 s}}\right)^{2}+\ldots\right) \\
& =\prod_{p}\left(\frac{1}{1-1 /\left(p p^{2 s}\right)}+\frac{p^{s} /\left(p p^{2 s}\right)}{1-1 /\left(p p^{2 s}\right)}\right) \\
& =\prod_{p}\left(1-\frac{1}{p^{2 s+1}}\right)^{-1}\left(1+\frac{1}{p^{s+1}}\right) .
\end{aligned}
$$


But

$$
\frac{\zeta(2 s)}{\zeta(s)}=\prod_{p}\left(1+\frac{1}{p^{s}}\right)^{-1}
$$

Hence

$$
\phi_{+}(s)=\frac{\zeta(2 s+1) \zeta(s+1)}{\zeta(2 s+2)} .
$$

Finally $\sigma+1>1,2 \sigma+1>1$ and $2 \sigma+2>1$ if $\sigma>0$.

Next, to derive the expression for $\psi_{-}(s)$ use

$$
\psi_{-}(s)=\sum_{n=1}^{\infty} \frac{r_{-}(n)}{n^{s}}=\sum_{n=1}^{\infty} \frac{1 / r_{+}(n)}{n^{s-1}}=\phi_{+}(s-1) .
$$

The other two derivations follow in a similar manner.

LEMMA 5.1.

$$
D_{2}(x, \alpha)=\sum_{1 \leq j \leq \alpha}\left\lfloor\frac{x}{r_{+}(j)}\right\rfloor \text {. }
$$

Proof. If $1 \leq j \leq \alpha$ and $j m=n^{2}$ for some $n \leq x$, let $j_{0}$ be such that $j j_{0}=n_{0}^{2}$ is the smallest multiple of $j$ which is a square: if

$$
j=\prod_{i=1}^{m} p_{i}^{\alpha_{i}}
$$

then

$$
j_{0}=\prod_{i=1}^{m} p_{i}^{\alpha_{i} \bmod 2}
$$

and $\left(\alpha_{i}+\alpha_{i} \bmod 2\right) / 2=\left\lceil\alpha_{i} / 2\right\rceil$ so $r_{+}(j)=n_{0}$.

Then

$$
D_{2}(x, \alpha)=\sum_{1 \leq j \leq \alpha} \sum_{\substack{j i=n^{2} \\ n \leq x}} 1=\sum_{\substack{1 \leq j \leq \alpha \\ j j_{0} m^{2}=n^{2} \\ n \leq x}} 1 .
$$

But $j j_{0} m^{2}=n^{2} \Leftrightarrow n_{0}^{2} m^{2}=n^{2} \Leftrightarrow n_{0} m=n, n \leq x \Leftrightarrow m \leq x / n_{0}$. Hence

$$
D_{2}(x, \alpha)=\sum_{1 \leq j \leq \alpha}\left\lfloor\frac{x}{r_{+}(j)}\right\rfloor
$$

EXAMPLE 5.1. An elementary derivation leads to an asymptotic formula for the partial sums of the squarefree reciprocals:

$$
F(x)=\sum_{\substack{1 \leq n \leq x \\ n \text { squarefree }}} \frac{1}{n}=\frac{\log x}{\zeta(2)}+O(1) .
$$


To see this, observe that

$$
\begin{aligned}
\sum_{1 \leq n \leq x} \mu(n)^{2} & =\sum_{1 \leq n \leq x} 1 \sum_{d^{2} \mid n} \mu(d)=\sum_{d \leq \sqrt{x}} \mu(d)\left\lfloor\frac{x}{d^{2}}\right\rfloor=x \sum_{d \leq \sqrt{x}} \frac{\mu(d)}{d^{2}}+O(\sqrt{x}) \\
& =x \sum_{d=1}^{\infty} \frac{\mu(d)}{d^{2}}+O(\sqrt{x})=\frac{6}{\pi^{2}} x+O(\sqrt{x}) .
\end{aligned}
$$

The result now follows by Abel's Theorem for partial summation [1].

A more precise result was found by Suryanarayana [13]. It is this form which we use in the restricted divisor derivation below, so we state it as a lemma.

LEMma 5.2 (Suryanarayana).

$$
F(x)=\frac{1}{\zeta(2)}\left(\log x+\gamma-2 \frac{\zeta^{\prime}(2)}{\zeta(2)}\right)+O\left(\frac{1}{\sqrt{x}}\right) .
$$

Theorem 5.2. For $x \rightarrow \infty$ and $1 \leq \alpha \leq x$,

$$
D_{2}(x, \alpha)=\frac{x \log ^{2} \alpha}{4 \zeta(2)}+\frac{x \log \alpha}{\zeta(2)}\left[\frac{3 \gamma}{2}-\frac{\zeta^{\prime}(2)}{\zeta(2)}\right]+O(x)+O(\alpha) .
$$

Proof. Using Lemma 5.1, we obtain

$$
D_{2}(x, \alpha)=\sum_{n \leq \alpha}\left\lfloor\frac{x}{r_{+}(n)}\right\rfloor=x \sum_{n \leq \alpha} \frac{1}{r_{+}(n)}+\sum_{n \leq \alpha}\left\{\frac{x}{r_{+}(n)}\right\}=x S(\alpha)+O(\alpha),
$$

where

$$
S(x)=\sum_{n \leq x} \frac{1}{r_{+}(n)} .
$$

Now let $x=y^{2}$ and for $d=1,2, \ldots$ let $S_{d}$ be the set of positive integers $n \leq x$ with largest squared factor $d^{2}$. Note that if $Q(x)$ is defined to be the set of squarefree integers less than or equal to $x$ then $S_{d}$ has $\left|Q\left(y^{2} / d^{2}\right)\right|$ elements. Note also that if $n$ is squarefree, then $r_{+}\left(n d^{2}\right)=n d$. Therefore

$$
\begin{aligned}
S\left(y^{2}\right) & =\sum_{d \leq y} \sum_{n \in Q\left(y^{2} / d^{2}\right)} \frac{1}{r_{+}\left(n d^{2}\right)}=\sum_{d \leq y} \sum_{n \in Q\left(y^{2} / d^{2}\right)} \frac{1}{n d} \\
& =\sum_{d \leq y} \frac{1}{d} \sum_{n \in Q\left(y^{2} / d^{2}\right)} \frac{1}{n}=\sum_{d \leq y} \frac{1}{d} F\left(\frac{y^{2}}{d^{2}}\right) .
\end{aligned}
$$

Now let

$$
\beta=\gamma-2 \frac{\zeta^{\prime}(2)}{\zeta(2)}
$$


and apply Lemma 5.2:

$$
\begin{aligned}
S(x)= & \sum_{n \leq \sqrt{x}} \frac{1}{n} F\left(\frac{x}{n^{2}}\right) \\
= & \frac{1}{\zeta(2)}\left[(\log x+\beta)\left(\sum_{n \leq \sqrt{x}} \frac{1}{n}-2 \sum_{n \leq \sqrt{x}} \frac{\log n}{n}\right)\right]+O\left(\frac{1}{\sqrt{x}} \sum_{1 \leq \sqrt{x}} 1\right) \\
= & \frac{1}{\zeta(2)}(\log x+\beta)\left(\log \sqrt{x}+\gamma+O\left(\frac{1}{\sqrt{x}}\right)\right) \\
& -\frac{2}{\zeta(2)}\left(\frac{1}{2} \log ^{2} \sqrt{x}+A+O\left(\frac{\log x}{\sqrt{x}}\right)\right)+O(1) \\
= & \frac{1}{4 \zeta(2)} \log ^{2} x+\frac{\log x}{\zeta(2)}\left(\frac{3 \gamma}{2}-\frac{\zeta^{\prime}(2)}{\zeta(2)}\right)+O(1)
\end{aligned}
$$

for some constant $A$. Therefore

$$
\begin{aligned}
D_{2}(x, \alpha) & =x\left[\frac{\log ^{2} \alpha}{4 \zeta(2)}+\frac{\log \alpha}{\zeta(2)}\left(\frac{3 \gamma}{2}-\frac{\zeta^{\prime}(2)}{\zeta(2)}\right)+O(1)\right]+O(\alpha) \\
& =\frac{x \log ^{2} \alpha}{4 \zeta(2)}+\frac{1}{\zeta(2)} x \log \alpha\left[\frac{3 \gamma}{2}-\frac{\zeta^{\prime}(2)}{\zeta(2)}\right]+O(x)+O(\alpha) .
\end{aligned}
$$

\section{Bounds for restricted sums for quadratic forms}

TheOREM 6.1. Let $f(n)$ be an irreducible polynomial with $f(n)>0$ for $n=1,2, \ldots$ If $1 \leq \alpha \leq x$ are real then there exist positive constants $c_{1}$ and $c_{2}$ such that

$$
c_{1} x \log \alpha \leq \sum_{1 \leq n \leq x} d_{\alpha}(f(n)) \leq c_{2} x \log \alpha .
$$

Proof. Define the three functions $\theta, \varrho$ and $N$ by

$$
\begin{aligned}
\theta(i, j) & = \begin{cases}1 & \text { if } i \mid f(j), \\
0 & \text { if } i \nmid f(j),\end{cases} \\
\varrho(i) & =\#\{j: i \mid f(j), 1 \leq j \leq i\}, \\
N(x, i) & =\#\{j: i \mid f(j), 1 \leq j \leq x\} .
\end{aligned}
$$

Then, since $i|f(j) \Leftrightarrow i| f(i+j)$ we have

$$
\left\lfloor\frac{x}{i}\right\rfloor \varrho(i) \leq N(x, i) \leq\left(\left\lfloor\frac{x}{i}\right\rfloor+1\right) \varrho(i)
$$

and so

$$
\frac{x}{2 i} \varrho(i) \leq N(x, i) \leq \frac{2 x}{i} \varrho(i) .
$$


Therefore

$$
\sum_{1 \leq i \leq \alpha} \frac{x}{2 i} \varrho(i) \leq \sum_{1 \leq i \leq \alpha} \sum_{1 \leq j \leq x} \theta(i, j) \leq \sum_{1 \leq i \leq \alpha} \frac{2 x}{i} \varrho(i)
$$

and so

$$
\frac{x}{2} \sum_{1 \leq i \leq \alpha} \frac{\varrho(i)}{i} \leq \sum_{1 \leq j \leq x} \sum_{1 \leq i \leq \alpha} \theta(i, j) \leq 2 x \sum_{1 \leq i \leq \alpha} \frac{\varrho(i)}{i}
$$

or

$$
\frac{x}{2} R(\alpha) \leq \sum_{1 \leq j \leq x} d_{\alpha}(f(j)) \leq 2 x R(\alpha)
$$

where

$$
R(x)=\sum_{1 \leq i \leq x} \frac{\varrho(i)}{i} .
$$

By Lemma 9 and Section 5 of [2],

$$
2 c_{1} \log x \leq R(x) \leq \frac{1}{2} c_{2} \log x
$$

and the conclusion of the theorem follows directly.

In case $f$ is quadratic, the previous result can be strengthened to give an asymptotic formula, using the results of McKee [8-10].

TheOREM 6.2. Let $f(n)=a n^{2}+b n+c$ be an irreducible quadratic polynomial with $f(n)>0$ for $n=1,2, \ldots$ Let $\Delta=b^{2}-4 a c<0$ not be a perfect square. If $1 \leq \alpha \leq x$ are real then there exists a positive constant $A_{f}$ such that

$$
\sum_{1 \leq n \leq x} d_{\alpha}(f(n))=A_{f} x \log \alpha+O(x)
$$

where

$$
A_{f}=\frac{6 H(\Delta)}{\pi \sqrt{-\Delta}} \prod_{p \mid a}\left(1-\frac{1}{p+1}\right),
$$

$H(\Delta)$ is the weighted class number (namely, the number of primitive and imprimitive forms $A x^{2}+B x y+C y^{2}$ with $B^{2}-4 A C=\Delta$, giving weight one half to forms proportional to $x^{2}+y^{2}$, and one third to those proportional to $\left.x^{2}+x y+y^{2}\right)$.

Proof. Using the same notation as in the previous theorem, it follows directly from (4) that

$$
N(x, i)=x \frac{\varrho(i)}{i}+O(\varrho(i)) .
$$

The same argument as that used in the theorem shows that

$$
\sum_{1 \leq n \leq x} d_{\alpha}(f(n))=x \sum_{1 \leq i \leq \alpha} \frac{\varrho(i)}{i}+O\left(\sum_{1 \leq i \leq \alpha} \varrho(i)\right) .
$$


Using the results of [10] given in Lemma 7 and Lemma 8 for the two sums in this formula, we obtain the result of the theorem with the given value for the constant $A_{f}$.

Acknowledgments. This work was done in part while the author was on study leave at Columbia University. The support of the Department of Mathematics at Columbia University and the valuable discussions held with Patrick Gallagher are warmly acknowledged. Helpful comments from a referee, especially those resulting in Theorem 6.2 , are also gratefully acknowledged.

\section{References}

[1] T. M. Apostol, Introduction to Analytic Number Theory, Springer, New York, 1976.

[2] P. Erdős, On the sum $\sum_{k=1}^{x} d(f(k))$, J. London Math. Soc. 27 (1952), 7-15.

[3] R. R. Hall and G. Tenenbaum, Divisors, Cambridge Univ. Press, 1988.

[4] C. Hooley, On the number of divisors of quadratic polynomials, Acta Math. 110 (1963), 97-114.

[5] M. N. Huxley, Area, Lattice Points and Exponential Sums, Oxford Univ. Press, 1996.

[6] A. E. Ingham, Some asymptotic formulae in the theory of numbers, J. London Math. Soc. (3) 2 (1927), 202-208.

[7] G. A. Kolesnik, An improvement in the remainder term in the divisor problem, Mat. Zametki 6 (1969), 545-554 (in Russian); English transl.: Math. Notes 6 (1969), 784-791.

[8] J. F. McKee, On the average number of divisors of quadratic polynomials, Math. Proc. Cambridge Philos. Soc. 117 (1995), 389-392.

[9] —, The average number of divisors of an irreducible quadratic polynomial, ibid. 126 (1999), 17-22.

[10] - A note on the number of divisors of quadratic polynomials, in: Sieve Methods, Exponential Sums, and their Application to Number Theory, London Math. Soc. Lecture Note Ser. 237, Cambridge Univ. Press, 1997, 275-281.

[11] Y. Motohashi, On the sum of the number of divisors in a short segment, Acta Arith. 52 (1970), 249-253.

[12] E. J. Scourfield, The divisors of a quadratic polynomial, Proc. Glasgow Math. Assoc. 5 (1961), 8-21.

[13] D. Suryanarayana, Asymptotic formula for $\sum_{n \leq x} \mu^{2}(n) / n$, Indian J. Math. 9 (1967), 543-545.

Department of Mathematics

University of Waikato

Hamilton, New Zealand

E-mail: kab@math.waikato.ac.nz 